\title{
НЕЙРОСЕТЕВОЙ АНАЛИЗ СОСТОЯНИЯ КОСТНОЙ ТКАНИ У ПАЦИЕНТОВ С САХАРНЫМ ДИАБЕТОМ
}

\author{
Сафарова С.С., Сафарова С.С.
}

Азербайджанский Медицинский Университет, Азербайджан, Баку

\begin{abstract}
ЦЕЛЬ: изучить предикторы связанные с ранними аберрационными изменениями в костной ткани у пациентов с сахарным диабетом 1 и 2 типа (СД1 и СД2) с помощью классической статистики и искусственной нейронной сети (ANN).
\end{abstract}

МАТЕРИАЛЫ И МЕТОДЫ: обследовано 98 пациентов С диабетом 1 типа (возраст 54,8ะ0,7 года), 137 -

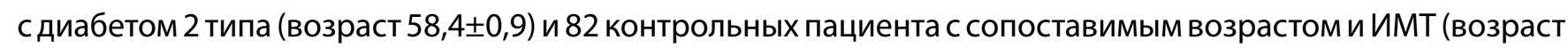
$55,9 \pm 0,9$ года). У всех пациентов были измерены ИМТ и минеральная плотность кости (МПК) по Т-критерию в поясничном отделе позвоночника (L1-4) и шейке бедренной кости (ШБ) методом двухэнергетической рентгенабсорбциометрии (DXA). Были определены $\mathrm{HbA}_{1 c^{\prime}}$ длительность диабета, сывороточные маркеры костного ремоделирования (P1NP и b-CTX), кальцитонин (KT), индекс НОМА-IR и СКФ.

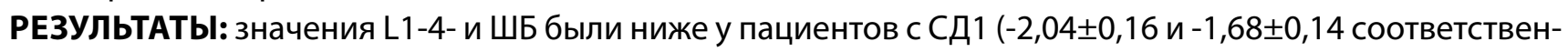

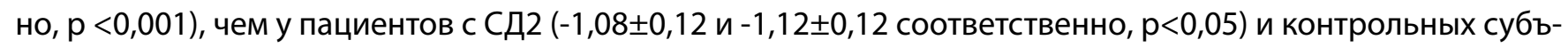

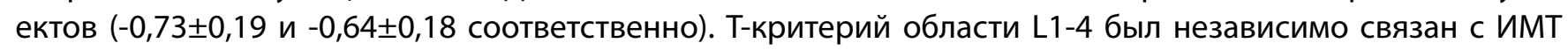
и $\mathrm{HbA}_{1 c^{\prime}}$ тогда как Т-критерий области ШБ был связан с ИМТ и КТ. У пациентов с СД2 выявлена значимая положительная зависимость между уровнем кальцитонина и индексом HOMA-IR ( $R=0,615 ; \mathrm{p}=0.03)$ возрастающая с увеличением длительности диабета. Значимая отрицательная связь определена между $\mathrm{HbA}_{1 c}$ и P1NP ( $R=-0,252 ; p=0,03$ для T1DM); $(R=-0,254 ; p=0,01$ для CД2). Отмечена значимая разница в уровне $\mathrm{b}-\mathrm{CTX}$ при СД в сравнении с контролем (p<0,05). Выявлена значимая отрицательная корреляция между уровнями альбумина и $b-C T x$ для $C Д 1(R=-0,330 ; p=0,01)$ и для $C Д 2(R=-0,387 ; p=0,001)$. Определена значимая отрицательная связь между СКФ и $b-C T x$ для CД1 ( $R=-0,204 ; p=0,04)$; для $C Д 2(R=-0,203 ; p=0,01)$.

Данные также анализировались разработанной программой с использованием системы поддержки принятия решений на базе ANN. Система на базе ANN выбрала 25 переменных для прогнозирования значений маркеров костного ремоделирования, T-критерия ШБ и L1-4 с точностью прогнозирования до $76-82 \%$ соответственно.

ВЫВОдЫ: настоящее исследование подтверждает, что низкая МПК в обеих зонах была косвенно связан с $\mathrm{HbA}_{1 c^{\prime}}$ через длительность течения диабета. При СД2 высокая МПК связана с повышенным ИМТ и низким КТ, при СД1 с высоким $\mathrm{HbA}_{1 с}$. Хронические осложнения негативно влияют на МПК. Важной частью использования ANN является процесс обучения, в котором данные неоднократно представляются в сеть, а обучение постепенно улучшает модель, для точности прогнозирования. Однако, когда анализ взаимосвязей между переменными в исследовании является сложным процессом, ANN могут быть эффективным решением, поскольку они обучаются, самостоятельно корректируют свою структуру и могут одновременно обрабатывать большое количество переменных.

КЛЮЧЕВЫЕ СЛОВА: диабетическая остеопатия; система поддержки принятия решений; искусственная нейронная cemb 